\title{
A new alternative in plant retrograde signaling
}

\author{
Hou-Sung Jung ${ }^{1}$ and Todd C Mockler ${ }^{2^{*}}$
}

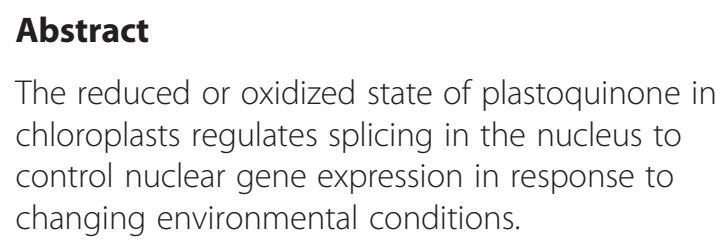

The reduced or oxidized state of plastoquinone in chloroplasts regulates splicing in the nucleus to control nuclear gene expression in response to changing environmental conditions.

\section{Introduction}

In plants, chloroplasts and the nucleus communicate with each other to regulate growth and adapt to environmental conditions. Signaling from the nucleus to chloroplasts, by which the nucleus programs chloroplast development, is called anterograde regulation. In signaling from chloroplasts to the nucleus, or retrograde signaling, the chloroplasts send signals to the nucleus for regulation of nuclear gene expression [1]. Similar to mitochondria, chloroplasts are semi-autonomous organelles. During their evolution, the chloroplasts relinquished most of their genetic information to the nucleus. Modern chloroplast genomes encode only about 100 proteins. Meanwhile, it is predicted that more than 3,000 nuclear genes encode chloroplast proteins. Thus, chloroplasts need to coordinate gene expression in both chloroplasts and the nucleus to conduct their biological functions. In addition, besides the chloroplast proteins, other functional proteins such as stress response proteins are also under the control of retrograde signaling. This indicates that chloroplasts are sensors that respond to changing environmental conditions by regulating nuclear gene expression.

Nuclear gene expression is regulated in multiple steps including transcription, mRNA maturation, and translation. It has recently been reported that the mRNA maturation step is involved in plant responses to environmental conditions [2]. As RNA-Seq technologies advance, we can expect that more interesting findings, especially related to transcript maturation, will be discovered. In Arabidopsis, more than $60 \%$ of intron-containing genes are alternatively spliced [3]. The frequency of alternative splicing (AS) further increases if different environmental conditions are

\footnotetext{
* Correspondence: tmockler@danforthcenter.org

²Donald Danforth Plant Science Center, St Louis, MO 63132, USA

Full list of author information is available at the end of the article
}

included. AS transcripts can have both positive and negative effects on nuclear gene expression [4]. AS events provide diverse transcripts (and eventually proteins) from a single gene. This provides plants with the potential for greater plasticity, which is critical for plants to deal with fluctuating environmental conditions. This positive function of AS is supported by recent results [5]. However, AS is not always productive. AS events include exon skipping, alternative splice site selection and intron retention [3]. Among them, the most abundant AS type in plants is intron retention [3]. Transcripts containing intron retentions are futile in many cases because of the inclusion of a premature termination codon [4]. Thus, it has been speculated that AS may occur erroneously rather than following a programmed instruction [4]. More studies are required to determine the biological functions of most AS events in plants.

\section{Reduced and oxidized states of plastoquinone}

Diverse chloroplast components have been proposed as the initiators of retrograde signaling. One of them is plastoquinone (PQ), a photosynthetic electron transport component [1]. PQ accepts electrons from the excited photosystem II (PSII) and becomes a reduced PQ $\left(\mathrm{PQH}_{2}\right) . \mathrm{PQH}_{2}$ travels through the thylakoid membrane to find the cytochrome $b_{6} f$ complex (cyt $b_{6} f$ ), a downstream component of PQ in the photosynthetic electron transport pathway. Following an electron donation to cyt $b_{6} f, \mathrm{PQH}_{2}$ is oxidized to $\mathrm{PQ}$.

The PQ's reduced or oxidized (redox) state rapidly changes depending on environmental conditions. For example, when the light intensity is too low, the PQ pool is in an oxidized state because less PQ is reduced to $\mathrm{PQH}_{2}$. In contrast, a high light intensity causes an imbalance between generation and utilization of NADPH in chloroplasts. In this condition, $\mathrm{PQH}_{2}$ is more abundant than PQ and the PQ redox state is a reduced one.

\section{$P Q$ redox state in retrograde signaling}

About 20 years ago, Escoubas et al. [6] reported for the first time that the PQ redox state initiates retrograde signaling in order to change nuclear gene expression in an 
alga. It was later reported that the reduced PQ pool regulates stress-related nuclear gene expression in Arabidopsis [7]. More recent genomic studies have demonstrated that the PQ redox state has a global effect on nuclear gene expression $[8,9]$. Previous research [1] had shown the retrograde regulation of nuclear gene expression mainly at the level of transcriptional control. Petrillo et al. [10] recently demonstrated that the $\mathrm{PQ}$ redox state regulates AS of RNA-processing protein genes including the ARGININE/ SERINE-RICH SPLICING FACTOR 31 (RS31) gene, which encodes a plant Serine/Arginine-Rich (SR) domain splicing factor. The authors observed that a dark condition inhibited RS31 mRNA maturation, leading to an increased level of RS31 AS transcripts. The inhibition of RS31 mRNA maturation was reversible because it was eliminated by light. After determining that RS31 AS events were independent from light quality and photoreceptors, Petrillo et al. [10] turned their attention to modulation of chloroplast functional states in the dark. Using 3-(3,4dichlorophenyl)-1,1-dimethylure (DCMU), which acts by blocking electron flow at the quinone acceptors of PSII, the authors showed that mRNA maturation was inhibited by the oxidized PQ state. Meanwhile, the reduced PQ state, which is induced by the chemical 2,5-dibromo-3methyl-6-isopropylbenzoquinone (DBMIB), could reverse the splicing inhibition events. The oxidized PQ state could reproduce the effects of dark on RS31 AS events, whereas the reduced PQ state resembled a light condition in terms of RS31 AS status. All of these results indicate that the light/dark shift-dependent RS31 AS is under the control of the PQ redox state. This is the first study reporting that the PQ redox state regulates the mRNA maturation steps for nuclear gene expression.

\section{Positive function of $R S 31$ alternative splicing}

The RS31 AS event reported by Petrillo et al. [10] is an example of a positive function of AS for gene expression regulation. In a light condition, RS31 transcript is fully spliced and generates $m R N A 1$, which encodes the functional RS31 protein. In a dark condition, however, AS generates $m R N A 2$ and $m R N A 3$, at the expense of $m R N A 1$. AS in the dark seems to be critical for this event because the authors determined that RS31 transcript levels were not significantly changed during the light-to-dark shift. It is unlikely that mRNA2 and mRNA3 encode a functional full-length RS31 protein or any of its subdomains. This is because both $m R N A 2$ and $m R N A 3$ contain a premature translation termination codon and because it was shown that most of these transcripts are retained in the nucleus. To understand the biological function of the dark-induced AS of RS31 from mRNA1 to mRNA2 and mRNA3, the authors generated a transgenic plant that constitutively expresses the RS31 mRNA1. The transgenic plant expresses a greater amount of the RS31 mRNA1, and is indistinguishable from wild-type plants in the light. In the dark, however, the over-accumulation of RS31 protein caused growth defect phenotypes. These results indicate that Arabidopsis uses AS to reduce the amount of RS31 protein levels to regulate plant development in light/dark shifts.

\section{Discussion}

The report by Petrillo et al. [10] will give momentum to forthcoming experiments in the retrograde signaling field. First, what are the signaling molecules that can travel from shoot-to-root as mentioned in their report? The authors showed that most of the previously reported signaling candidate molecules, including reactive oxygen species, are unrelated to this PQ-dependent splicing event. Therefore, molecules that trigger this event remain to be discovered. Second, what intron-splicing components are regulated by the PQ redox state? Intron splicing from pre-mRNA requires many protein components. It would be interesting to investigate what components sense the PQ redox state in the nucleus. Third, a number of nuclear genes are under the control of the PQ redox state $[8,9]$. Do any of them undergo AS when they are induced by the PQ redox state? It is especially worth investigating the splicing variants of nuclear genes that are significantly induced by the PQ redox state. Finally, it would be interesting to know whether RS31 AS will also be observed when the PQ redox state is induced by a shift between PSII- and PSI-specific light conditions [8]. In summary, Petrillo et al. [10] reported interesting phenomena that connect plants' responses to changing light environments with the PQ redox state and AS events. Further studies will show how the PQ redox-driven retrograde signaling from chloroplasts regulates AS events in the nucleus. Ultimately, these findings can be applied to generate crop plants with enhanced adaptation to environmental conditions including abiotic stresses.

\section{Abbreviations \\ AS: Alternative splicing; NADPH: Reduced form of $\mathrm{NADP}^{+}$(nicotinamide adenine dinucleotide phosphate); PQ: Plastoquinone; redox: reduced or oxidized.}

\section{Competing interests}

The authors declare that they have no competing interests.

\section{Author details}

${ }^{1}$ Biological Sciences, Dartmouth College, Hanover, NH 03755, USA. ²Donald Danforth Plant Science Center, St Louis, MO 63132, USA.

Published: 30 May 2014

\section{References}

1. Pfalz J, Liebers M, Hirth M, Gruebler B, Holtzegel U, Schröter Y, Dietzel L, Pfannschmidt T: Environmental control of plant nuclear gene expression by chloroplast redox signals. Front Plant Sci 2012, 3:257. 
2. Posé $D$, Verhage L, Ott F, Yant L, Mathieu J, Angenent GC, Immink RGH, Schmid M: Temperature-dependent regulation of flowering by antagonistic FLM variants. Nature 2013, 503:414-417.

3. Syed NH, Kalyna M, Marquez Y, Barta A, Brown JWS: Alternative splicing in plants - coming of age. Trends Plant Sci 2012, 17:616-623.

4. Mastrangelo AM, Marone D, Laidò G, De Leonardis AM, De Vita P: Alternative splicing: Enhancing ability to cope with stress via transcriptome plasticity. Plant Sci 2012, 185-186:40-49.

5. Staiger D, Brown JWS: Alternative splicing at the intersection of biological timing, development, and stress responses. Plant Cell 2013, 25:3640-3656.

6. Escoubas J-M, Lomas M, LaRoche J, Falkowski PG: Light intensity regulation of cab gene transcription is signaled by the redox state of the plastoquinone pool. Proc Natl Acad Sci USA 1995, 92:10237-10241.

7. Karpinski S, Reynolds H, Karpinska B, Wingsle G, Creissen G, Mullineaux P: Systemic signaling and acclimation in response to excess excitation energy in Arabidopsis. Science 1999, 284:654-657.

8. Fey V, Wagner R, Braütigam K, Wirtz M, Hell R, Dietzmann A, Leister D, Oelmüller R, Pfannschmidt T: Retrograde plastid redox signals in the expression of nuclear genes for chloroplast proteins of Arabidopsis thaliana. J Biol Chem 2005, 280:5318-5328.

9. Jung H-S, Crisp PA, Estavillo GM, Cole B, Hong F, Mockler TC, Pogson BJ, Chory J: Subset of heat-shock transcription factors required for the early response of Arabidopsis to excess light. Proc Natl Acad Sci USA 2013, 110:14474-14479.

10. Petrillo E, Herz MAG, Fuchs A, Reifer D, Fuller J, Yanovsky MJ, Simpson C, Brown JWS, Barta A, Kalyna M, Kornblihtt AR: A chloroplast retrograde signal regulates nuclear alternative splicing. Science 2014, 344:427-430.

doi:10.1186/gb4178

Cite this article as: Jung and Mockler: A new alternative in plant retrograde signaling. Genome Biology 2014 15:117. 